\title{
Towards Operational Systems for Continuous Navigation of Rescue Teams
}

\author{
Michael Angermann, Mohammed Khider, Patrick Robertson \\ Institute of Communications and Navigation \\ German Aerospace Center (DLR) \\ P.O. Box 1116, D-82234, Wessling, Germany \\ E-mail: \{firstname.lastname\}@dlr.de
}

\begin{abstract}
Rescue workers, such as fire fighters often face conditions that impose a combination of threats on them: severe physical and mental stress, limited or no visibility, unknown layout of buildings may induce disorientation. Systems capable of continuously locating all members of a rescue team could help to maintain orientation, and in cases of accident, help other team members or team leaders to localize colleagues in danger. The paper points out the discrepancy between state of the art in research projects, primarily in the fields of wearable computing and indoor navigation, and the lack of operational systems that have been fielded or have undergone sustained usage. The paper shows, that a successful operational system for continuous navigation of rescue teams requires a combination of several sensors, motion models, computing, communication means, and human machine interfaces. Based on an analysis of rescue operations and interviews with rescue workers, relevant issues and requirements are derived. A reference architecture for an operational system for continuous navigation of rescue teams is outlined. Its implementation and feasibility aspects are discussed. We discuss in how far the required technological components are available and affordable and where shortcomings have to be resolved by future research and development.
\end{abstract}

\section{INTRODUCTION}

During search and rescue operations self-protection of rescue teams has absolute priority over all other objectives. Nevertheless rescue workers, such as fire fighters often face conditions that impose a combination of threats on them: severe physical and mental stress, limited or no visibility, unknown layout of buildings may induce disorientation, which, in combination with excessive heat, toxic gases, fumes or other substances, can turn a rescue operation into a tragedy. An upsetting example is given in [1]:

On December 3, 1999 six fire fighters lost their lives in a tragic cold storage warehouse fire in the City of Worcester, Massachusetts. Two fire fighters initially got lost and then two search teams also became lost in the maze and zero visibility from the dense smoke resulting in the six deaths.

Obviously, a system capable of continuously locating all members of a rescue team could help a rescue worker to maintain orientation, and in cases of accidents, help other team members or operations controllers to localize colleagues in danger. Several research projects, primarily in the fields of wearable computing and indoor navigation, have taken the described application as their reference case. However, few operational systems have been fielded or have undergone sustained usage.

This paper aims at pinpointing relevant issues for further research and development in order to achieve fieldable systems as soon as possible. The paper is structured as follows: We give a short overview over several research projects as an account of the state of the art in research (II-A). Based on an analysis of rescue operations and interviews with rescue workers we show the state of the art in daily operations (II-B) and discuss issues and requirements that should be taken into account by any system that aims to become operational (III-A). These general issues are then discussed in the light of the individual subsystems, i.e. localization (III-B), communication (III-C), mapping (III-D) and user interface (III-E). We will then briefly outline a reference architecture for an operational system for continuous navigation of rescue teams and discuss its implementation and feasibility aspects (IV). We will then conclude and show in how far the required technological components are available and affordable and where shortcomings have to be resolved by future research and development (V). We hope that our findings can contribute to guideline the future research in fields, such as motion modelling, sensor fusion, or location visualization, towards advances that, eventually, will lead to operational systems that will improve the safety, efficiency and effectiveness of rescue workers.

\section{StAte OF The ART}

\section{A. Research}

An increasing amount of research effort is being dedicated to support reliable location determination and joint situation awareness of firefighters. The following list of research projects gives an overview of the state of the art in research. This list does not claim to be comprehensive and is likely to have a European bias. 
1) IP Firefighter: The project IP Firefighter was mainly driven by the idea of networking firefighters with each other (the acronym "IP" refers to the Internet Protocol used for networking in this project). IP Firefighter was carried out in Denmark by TDC Mobil and Systematic Software Engineering $\mathrm{A} / \mathrm{S}$ and included the Fire Services of Aarhus and Copenhagen and the ambulance service Falck.

The prototype developed in IP Firefighter uses a standard commercial wireless network (Wireless LAN 802.11) for networking individual firefighters and their team leader via an access point. The individual firefighters are equipped with GPS, a sensor for measuring electrocardiograms (ECGs), body temperature and a computer with a module for Wireless LAN fitted in the breast pocket of their jackets. An application on a tablet PC provides administrative overview to the team leader and sends automatic alarms if a firefighter is in difficulty.

The prototype allows only outdoor location determination from GPS. Indoor location determination as well as extending the system's stamina (current battery lifetime is stated to be less than half an hour) is foreseen for future improvements by the consortium [2].

2) PeLoTe: PeLoTe (Building Presence through Localization for Hybrid Telematic Systems) is funded by the European Commission under the IST programme. The project has a strong focus on the interaction between humans and robots, but is considered relevant in the context of navigation for rescue teams, due to fairly extensive work on indoor navigation and support for firefighters as an application scenario.

PeLoTe developed the Personal Navigation system (PeNa) for indoor localization of humans. The system relies on the fusion of various sensors, such as inertial sensors, laser scanners and GPS. Furthermore, PeLoTe has performed work on standardized formats for mapping (SRM - Standard Rescue Map) and Simultaneous Localization and Mapping (SLAM) [3].

3)wearIT@WORK: wearIT@WORK is an ongoing project in the area of wearable computing and also funded by the European Commission under the IST programme. One of the four application scenarios of wearIT@WORK is emergency response. The project performs user trials with the Paris Fire Brigade. The integrated use of map information and localization sensors that do not require infrastructure, such as shoe-mounted inertial sensors is studied. Similar to [4], wearIT@WORK uses particle filtering algorithms to perform sensor fusion [5].

4) Precision Personnel Locator (PPL): The Precision Personnel Locator (PPL) is being developed by Worcester Polytechnic Institute, Massachusetts for supporting first responders. The project is funded by multiple sources, namely the Office of Science and Technology, the National Institute of Justice and the Federal Emergency Management Agency (FEMA) Fire Prevention and Safety. In contrast to most of the European projects, PPL employs dedicated transmitters in badges worn by firefighters. These badges continuously emit OFDM (Orthogonal Frequency Division Multiplex) modulated radio signals. Receivers for these signals are mounted on fire trucks. Given the knowledge of the fire trucks' positions, the locations of the firefighters can be computed from the time-of-fight measurements [6], [7].

5) DLR Advanced Navigation Applications: Within its strategic research program for advanced navigation applications, German Aerospace Center develops an indoor localization system. The system's core is a particle-filter based sensor-fusion engine. The system is capable of utilizing information coming from a wide range of sensors, such as GPS, shoe-mounted inertial sensors, magnetometers, barometers, RFID-tags as well as a priori information from maps and movement models [4], [8].

\section{B. Operational Systems}

Firefighters that enter the burning structure always face a risk of being trapped inside and may be in need of assistance themselves, but not be able to call for help. In order to avoid that these firefighters are left unnoticed without help the ingress and egress of firefighters from the scene is monitored. The typical tool for this is a chalkboard, grease pencil board, or prefabricated tags with the name of the firefighters on a structured board (see Fig. 1).

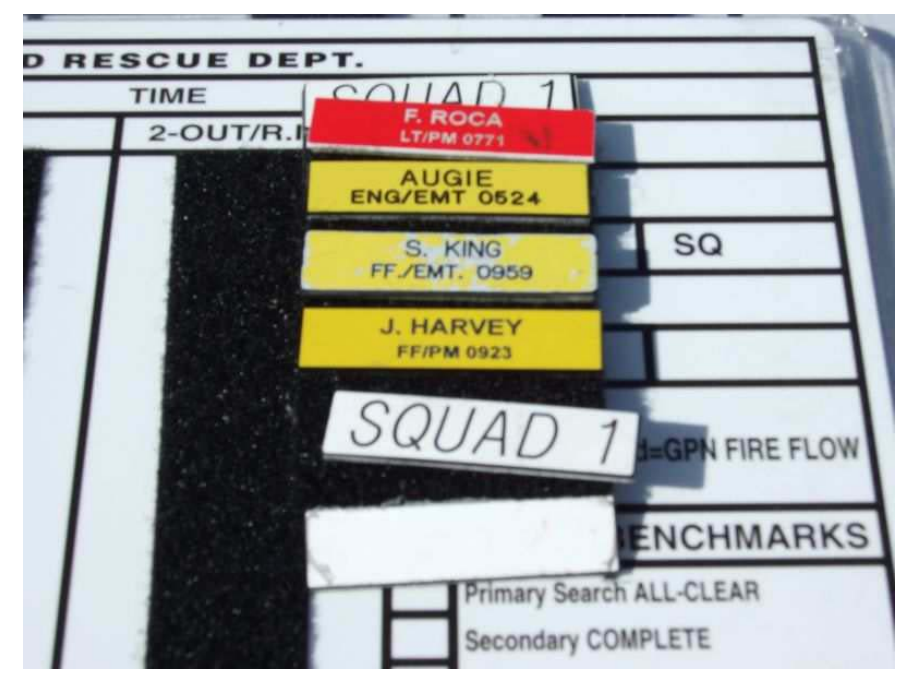

Fig. 1. Board for tracking the status of firefighters and the fire scene. Prefabricated name-tags are attached to the board to keep track of the firefighters at the scene.

The tags are typically either magnetic or fitted with velcro tape to stay in place. A number of systems that replicate this functionality on a laptop are also in use with some fire departments.

Fire scenes are generally very dynamic places with rapidly changing situations and a large number of involved personnel. 
It therefore happens that firefighters enter or leave the scene without being correctly accounted for. These two cases may result in two distinguishable, but equally dangerous effects: Firstly, when an unaccounted firefighter experiences problems without being able to call for help, his absence will be unnoticed and no help can be sent. Secondly, when a firefighter leaves the scene without his state being changed on the monitoring board, this will lead to a secondary search and rescue operation for the supposedly missing firefighter when his predefined safe-return time has elapsed. This secondary search and rescue operation then exposes the participating firefighters to significant and needless risk.

It is obvious that these two problems could be avoided if a system for precisely determining the location of every firefighter on the scene would be in use. Furthermore, this would virtually eliminate the time for the "search", thereby increasing the likelihood of successful "rescue" in the aforementioned secondary search and rescue operation. However, we have not found even rudimentary forms of such systems to be used by fire brigades in their daily operations.

\section{IsSUES, REQUIREMENT AND APPROACHES}

Despite the fact that several research projects have achieved good results in indoor localization, considerable effort is required to forward the current prototypes towards fieldable systems that can be used in daily operations under all occurring conditions. In this section we will discuss some of the partially open issues and requirements for viable system and will discuss approaches for realization of the subsystems for localization, communication, mapping and user interfacing.

\section{A. Overall System}

The following list may be used as a high-level checklist or work programme for the development of operational systems for continuous navigation of rescue teams:

1) indoor and outdoor

2) $2 \mathrm{D}$ and $3 \mathrm{D}$

3) joint situation awareness

4) accuracy

5) autonomy

6) lightweight and unobtrusive

7) stamina

8) robustness

9) economical feasibility

It is important to address all these issues within one integrated system. It is hardly conceivable that a fire department will procure, maintain and train on multiple systems.

The system has to work both indoor and outdoors and has to cope with fast transients between indoor and outdoor.
Full 3D capability has to be achieved. It has to be taken into account that rescue teams may not always use stairs, but may be brought up by hydraulic ladders or use ropes to descent.

Information on positions has to be provided to all involved persons, such as team member or team leader, in order to provide joint situational awareness. This requires reliable communication means for exchanging the location data.

The system has to be as accurate as possible to narrow down the search space in case of accidents as much as possible. However, we have to be aware that the accuracy will not be constant but a function of space and time. It is therefore important to communicate the actual uncertainty to the users. The system has to be able to work for a few hours without exchange of batteries and should ideally work independently of external (potentially damaged) infrastructure (with GPS being an exemption).

The system has to be robust against environmental conditions, such as heat, water, or mechanical shock.

Even if all the previous issues are solved to satisfaction, a system will fail to unfold its benefits if its cost prevents procurements by fire brigades. From a research perspective, we easily assume that no cost is too high for saving lives. However, economic aspects do play a role and economic feasibility is crucial.

\section{B. Localization Subsystem}

The requirement to work both indoors and outdoors, with fast transients in between, causes particular challenges for initial alignments and position fixes for the inertial navigation component. It is not feasible to perform extended "calibration walks" when arriving at the scene. However, the requirement to support potential operations in structures with reenforced concrete or even underground cannot be fulfilled by GNSS alone. A successful operational system for continuous navigation of rescue teams can hardly be based on one sensor alone, but requires a combination of several sensors and motion models. Figure 2 shows an overview of the realistically available and affordable sensors that have to be integrated by suitable fusion algorithms. An interesting additional source of location information may be rapidly deployable radio transmitter systems such as described in [6], [7]. In order to work in difficult underground areas, such radio-based systems may have to include relay nodes. These relay nodes have to determine their own positions and then act as transmitters with known positions themselves.

\section{Communication Subsystem}

Since location information has to be forwarded to all involved team members a suitable wireless data communication subsystem has to facilitate data exchange. It has to be taken 


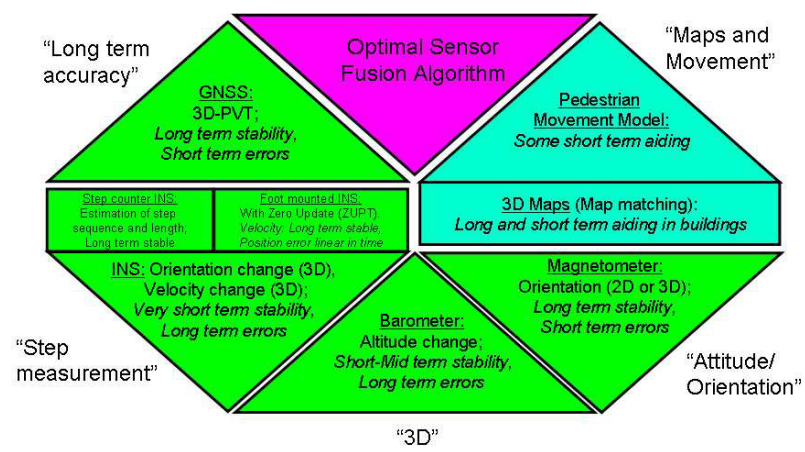

Fig. 2. Components of localization subsystems. Sensors (green), a priori knowledge (cyan), and sensor fusion algorithms (magenta) have to be tightly integrated for achieving the required capabilities.

into account that the radio propagation channel may be exhibit strong attenuation (e.g. from ground level to an underground metro station). For this reason adhoc networking between adjacent team members and possible relay nodes is considered advantageous.

\section{Mapping Subsystem}

The use of mapping information in indoor navigation systems is twofold: Firstly, maps are important help the user in interpreting the positions with respect to the structure of a building. Additional valuable information, such as the location of hazardous material is well representable on maps. Secondly, map information is used as a priori knowledge to improve the position estimates of advanced sensor fusion algorithms, such as particle filters and can significantly increase accuracy and stability of the location estimate. However, it is important to take possible map errors into account. Such map errors may be missing walls or falsely assumed walls where there are none. Since, such map errors are inevitable, a mapping system has to be able to express the uncertainty of its data base.

A difficult issue is the availability of digital building floor plans. Such floor plans should be stored centrally in databases and made accessible via wireless data communication. Additionally, the should be locally stored at buildings, and pre-stored on the navigation system. Sufficient redundancy should be achievable by this combination.

While some projects investigate the online generation of maps, e.g. by laser scanners, this approach may still need further research before becoming robust enough for daily operations.

\section{E. User Interface Subsystem}

In most tracking systems, the position of a tracked entity (e.g. a firefighter) is shown to the user by placing a symbol at the supposed position of the tracked entity on a map. However, in the light of the significant uncertainties in the estimated positions in indoor environments, it is necessary to communicate the remaining uncertainty to the user. While some systems provide some information on the uncertainty, e.g. in the form of error ellipses, we believe that due to the multi-modality (see Fig. 3) of the position estimate, it is beneficial to explicitly visualize the probability density function (PDF) of the position estimate to the user. Decisions, such as how many firefighters to send on a secondary search and rescue mission, can be taken much more informed, if a team leader can see that the location estimate indicates that the missing person may be in in one of several different rooms with almost identical probability.

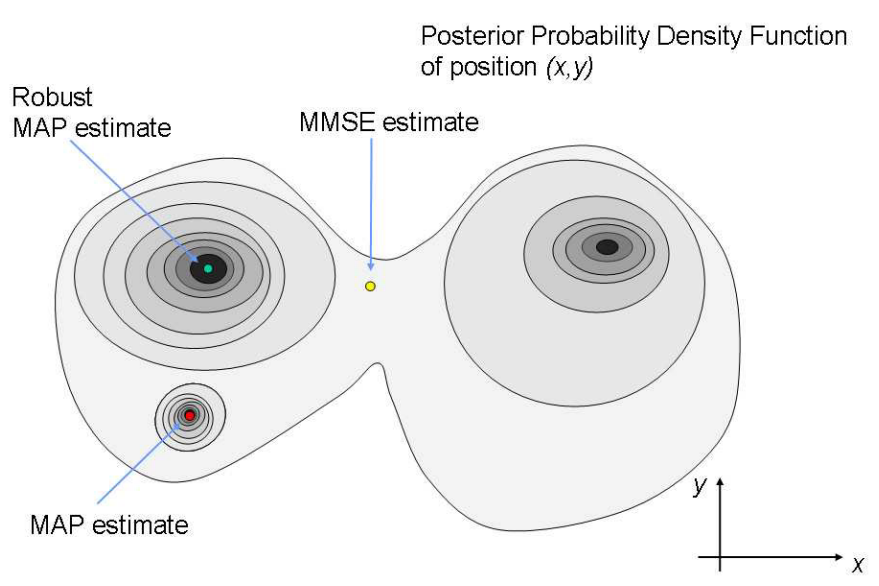

Fig. 3. Inherent uncertainties in location estimates should be explicitly communicated to the user. The multi-modal character of the position estimates is best expressed by visualizing the entire probability density function (PDF). Point estimates, such as MAP (maximum a posteriori) or MMSE minimum mean square error are often misleading,

\section{High LeVel Reference Architecture}

A high level reference architecture that includes the subsystems for localization/context awareness, mapping, user interface and communication and their interplay is illustrated in Fig. 4.

As mentioned before, the need to distribute the localization information to all involved entities and the potentially difficult radio propagation conditions for both communication and navigation, call for a adhoc communication between the involved nodes (see Fig. 5 for illustration).

\section{CONCLUSiOnS AND OUTLOOK}

Several core technologies are required for continuous navigation systems for rescue teams. Various research projects have contributed to significant advances in these core technologies: Advanced sensor fusion algorithms, such as particle filtering for localization are already providing promising accuracy and availability. Moreover, these algorithms are capable to achieve good results with relatively inexpensive sensors, such as consumer-grade GPS receivers and MEMS-based accelerometers and gyroscopes. The 
proliferation of wireless data communication into massmarket consumer products has the positive side-effect of rendering highly integrated wireless modules, required for the exchange of sensor and location data, available at almost no cost.

Most of the mentioned research projects have invested considerable effort in investigating the operational scenarios of their potential users and have thereby obtained a better understanding of the resulting system requirements. As many of the involved researchers acknowledge, this has significantly changed their perception of the problem and has opened many new, crucial and open issues during the course of their projects. As a result, it has become clear that significant work on a number of these crucial issues is still necessary before we will see the successful transfer of the numerous
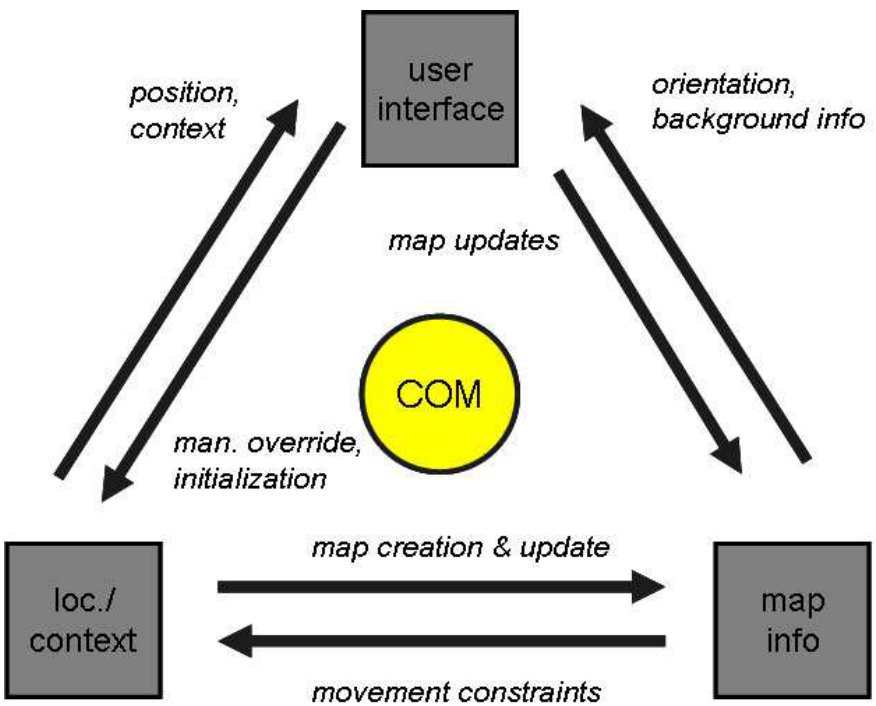

Fig. 4. High level architecture for continuous navigation system (interplay of subsystems).

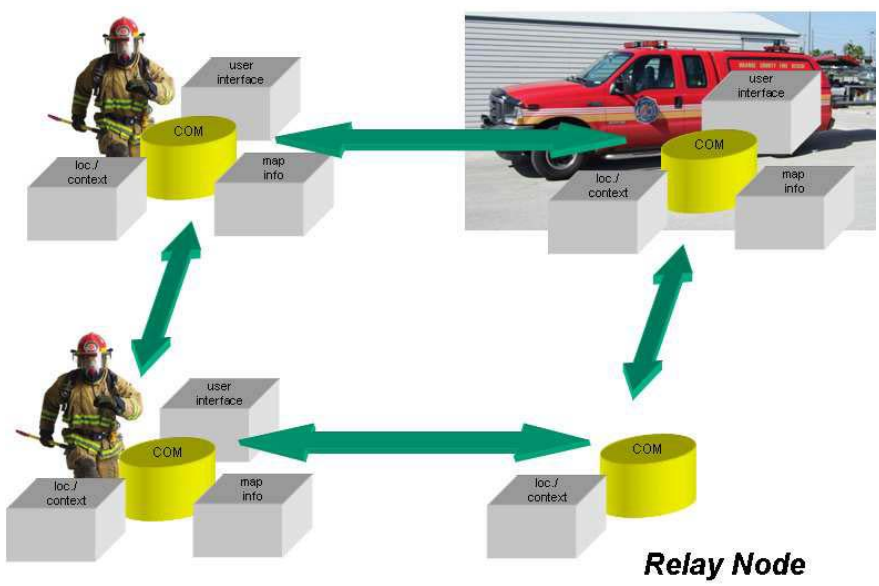

Fig. 5. High level architecture for continuous navigation system (interplay of nodes). experimental prototypes into daily operational use.

Probably the most relevant of these issues are related to the "imperfectness" of the real world compared to simulations or controlled lab environments: We see a strong need to study and cope with the lack of maps that represent the interior of buildings or underground structures. Closely related to this issue are map errors. While the sensor fusion algorithms need maps to provide long-term stability, they are particularly susceptible to map errors. Since map errors are inevitable, we have to find ways to deal with them by properly representing and taking the uncertainties in maps into account.

In contrast to the majority of conventional pedestrian navigation systems, navigation systems for supporting rescue teams have to maintain good position estimates even during phases of movements other than walking, such as crawling or climbing.

Since we doubt that the potential position error can be narrowed down to few centimeters under all conditions, we strongly advocate for internally representing the uncertainty of the location estimation and to communicate this uncertainty via the user interface. This may be achieved by color-coded or contour plots of the probability density of the position instead of point estimates as overlays on top of building plans or floor layouts. This is particular important due to the existence of multimodal probability densities that, e.g. in case of accidents, may be used to decide to send help to more than one room in parallel. Hence, subjective evaluation of performance under an exhaustive set of test scenarios is at least as important as objective performance indicators such as mean squared position error which are primarily meaningful to evaluate point estimators.

The existing issues are challenging and require further investigation and engineering. Nevertheless, we consider the development of systems that fulfill the requirements to be feasible. We are therefore optimistic and believe that in the not too distant future the research effort will result in operational systems that will indeed improve the safety and effectiveness of rescue workers.

\section{ACKNOWLEDGEMENT}

We are deeply indebted to all the members of professional fire brigades and volunteer fire companies in all ranks, that took the time to explain and show us the real-world issues and problems of their profession.

\section{REFERENCES}

[1] J. A. Orr and D. Cyganski, "Personnel location technology for incident response," Worcester Polytechnic Institute," Whitepaper, 2001.

[2] IP Firefighter, "IP Firefighter," Systematic Software Engineering A/S," Whitepaper, 2003.

[3] PELOTE, "Deliverable 2.1: Research analysis and design of algorithms and sensors for personal navigation system PENA," Project PELOTE, IST-2001-38873," Whitepaper, 2003. 
[4] K. Wendlandt, M.Khider, M.Angermann, and P.Robertson, "Continuous location and direction estimation with multiple sensors using particle filtering," in IEEE International Conference on Multisensor Fusion and Integration for Intelligent Systems, Germany, 2006.

[5] S. Beauregard, "Omnidirectional pedestrian navigation for first responders," in Proceedings of the 4th Workshop on Positioning, Navigation and Communication (WPNC 2007), Hannover, Germany, 2007.

[6] D. Cyganski, J. Duckworth, S. Makarov, W. Michalson, and J. Orr, "WPI Precision Personnel Locator System - Evaluation by First Responders," in The 20th International Technical Meeting of the Institute of Navigation Satellite Division, Fort Worth, TX, 2007.

[7] D. Cyganski, J. Duckworth, S. Makarov, W. Michalson, J. Orr, et al., "WPI Precision Personnel Locator System - Indoor Location Demonstrations and RF Design Improvements," in Institute of Navigation, 63rd Annual Meeting, Cambridge, MA, 2007.

[8] M.Khider, S.Kaiser, P.Robertson, and M.Angermann, "A Novel Movement Model for Pedestrians Suitable for Personal Navigation," in ION NTM, San Diego, USA, Jan. 2008. 